BULL. AUSTRAL. MATH. SOC.

VOL. 14 (1976), 405-408.

\title{
On the invariance of certain estimators
}

\section{J.E. Gentle and V.A. Sposito}

In this note, $L_{p}$ estimators for the parameters in the linear model $y=X \beta$ are considered. In particular, it is shown that these estimators are invariant under scale transformations on the dependent variable; that is, if $\hat{\beta}(y, X)$ is an $L_{p}$ estimator for $\beta$, then $a \hat{\beta}(y, X)=\hat{\beta}(\alpha y, X)$ for any nonzero scalar $a$. It is shown that this result does not extend to more general transformations on $y$, and the invariance property does not hold for general nonlinear models.

\section{Invariance}

Computing $L_{p}$ estimators by linear functions on discrete data has received much attention in the literature in the past few years; recent articles have appeared for determining $L_{p}$ estimators when $p=1,2$, and - (see Appa and Smith [1], Barrodale and Roberts [2], and Wagner [9]). The $L_{p}$-criteria for various values of $p$ have been discussed by Barrodale and Roberts [3], Barrodale, Roberts, and Hunt [4], Ekblom and Henriksson [5], Forsythe [6], and Rice and White [8]. Several properties of $L_{1}$ and $L_{\infty}$ estimators are given in [1]; in [3] some properties of $L_{p}$ estimators are established. This note will address itself to another property of any $L_{p}$ estimator (for any $p \neq 0$ ). In particular, it is shown that such estimators are invariant under scale transformations on the dependent

Received 17 February 1976. 
variable. The concept of invariance is discussed by Fraser [7, p. 67] and is defined for scale transformations as:

DEFINITION. $\hat{\beta}(y, X)$ is an invariant estimator for $B$ if

$$
a \hat{\beta}(y, x)=\hat{\beta}(a y, x) \text {. }
$$

The following lemma shows that under the $L_{p}$-criteria for any $p(\neq 0)$ the $L_{p}$ estimator, $\hat{B}(y, x)$, is invariant in the case of the linear model.

LEMMA. If $\underset{\beta}{\min }\|y-X \beta\|^{p}=\|y-X \hat{\beta}\|^{p}$, then for any scalar $a \neq 0$,

(i) $\underset{\delta}{\min }\|a y-X \delta\|^{p}=\|a\|^{p}\|y-X \hat{\beta}\|^{p}$, and

(ii) $\hat{\delta}=a \hat{\beta}$.

Proof. If $\underset{\beta}{\min }\|y-X \beta\|^{p}=\|y-X \hat{\beta}\|^{p}$, then

$$
\begin{aligned}
\min _{\delta}\|a y-X \delta\|^{p} & =\|a\|^{p} \underset{\delta}{\min }\left\|y-\frac{1}{a} x \delta\right\|^{p} \\
& =\|a\|^{p} \underset{\beta}{\min }\|y-X \beta\|^{p},
\end{aligned}
$$

where

$$
\begin{aligned}
\beta & =\frac{1}{a} \delta \\
& =\|a\|^{p}\|y-X \hat{\beta}\|^{p},
\end{aligned}
$$

and the result follows.

\section{Summary and examples}

When $p=1,2$, and $\infty$, the best $L_{p}$ estimators of the location parameter in the model $y=\beta_{0}$ correspond to the median, mean, and midrange, respectively $([1],[5],[7]$, and [8]). Hence, appealing to the lemma, for any scalar $a(\neq 0)$,

$$
a y_{M}, a \bar{y} \text {, and } a y_{M R}
$$


are optimal under $L_{1}, L_{2}$, and $L_{\infty}$, respectively.

Consider the model $y=\beta^{3}$. This model is nonlinear with respect to the unknown parameter, $\beta$. Under the $L_{2}$-criterion

$$
\hat{\beta}=(\bar{y})^{1 / 3} \text {, }
$$

and for any scalar $a(\neq 0)$,

$$
\hat{\beta}^{*}=(a \bar{y})^{1 / 3} \text {. }
$$

Hence, the estimator is not invariant, but is invariant under the transformation $\delta=\beta^{3}$. In this case,

$$
\hat{\bar{\delta}}=\bar{y} \text { and } a \hat{\bar{\delta}}=a \bar{y} \text {. }
$$

When the nonlinear model is separable with respect to the unknown parameters, then the corresponding $L_{p}$ estimator is invariant in view of the above transformation. The invariance property of the $L_{p}$ estimators does not hold for general nonlinear models. For example, consider the model

$$
y=\beta_{1} e^{-\beta_{2} x}
$$

then for any optimal $L_{p}$ estimator

$$
\tilde{\beta}=\left(\tilde{\beta}_{1}, \tilde{\beta}_{2}\right)
$$

and any $a(\neq 0)$,

$$
\tilde{\bar{B}}=\left(a \tilde{\beta}_{1}, \tilde{\beta}_{2}\right)
$$

In [4], models nonlinear in one parameter are discussed; for these models, the estimators are not invariant. Examples of these models are:

$$
\begin{aligned}
& y=\left(\beta_{0}+\beta_{1} x\right) /\left(1+\beta_{2} x\right), \\
& y=\beta_{0}+\beta_{1} /(1+x)^{\beta_{2},}
\end{aligned}
$$

and 


$$
y=e^{\beta_{2} x}\left(B_{0}+\beta_{1} x\right)
$$

\section{References}

[1] Gautam Appa and Cyril Smith, "On $L_{1}$ and Chebyshev estimation", Math. Programming 5 (1973), 73-87.

[2] 1. Barrodale and F.D.K. Roberts, "An improved algorithm for discrete $l_{1}$ linear approximation", SIAM J. Numer. Anal. 10 (1973), 839-848.

[3] I. Barrodale and F.D.K. Roberts, "Applications of mathematical programming to $\tau_{p}$ approximation", Nonlinear programming, 447-464 (Proc. Sympos. Mathematics Research Center, University of Wisconsin, Madison, 1970. Academic Press, New York, London, 1970).

[4] I. Barrodale, R.D.K. Roberts and C.R. Hunt, "Computing best $\tau_{p}$ approximations by functions nonlinear in one parameter", Comput. J. $13(1970), 382-386$.

[5] H. Ekblom and S. Henriksson, " $L_{p}$-criteria for the estimation of location parameters", SIAM J. Appl. Math. 17 (1969), 1130-1141.

[6] Alan B. Forsythe, "Robust estimation of straight line regression coefficients by minimizing pth power deviations", Technometrics 14 (1972), 159-166.

[7] D.A.S. Fraser, Nonparametric methods in statistics (John Wiley \& Sons, New York, London, Sydney, 1957).

[8] John R. Rice and John S. White, "Norms for smoothing and estimation", SIAM Rev. 6 (1964), 243-256.

[9] Harvey M. Wagner, "Linear programing techniques for regression analysis", J. Amer. Statist. Assoc. 54 (1959), 206-212. 